\title{
Os antecedentes e as dimensões do brand equity: um estudo das marcas de lojas de vestuário na cidade de Fortaleza
}

\section{The antecedents and dimensions of brand equity: a study on brands of clothing stores in Fortaleza city}

\author{
José Sarto Freire Castelo 1 \\ Arnaldo Fernandes Matos Coelho ${ }^{2}$ \\ José Ednilson de Oliveira Cabral ${ }^{3}$
}

\section{Resumo}

Este artigo avalia as relações entre os elementos do marketing mix e a criação de brand equity, seguindo um modelo conceitual definido a partir da literatura de marketing. O estudo é baseado nas percepções individuais de 571 clientes selecionados no contexto de um top of mind assistido de oito marcas de lojas de Fortaleza. Os resultados dos testes das hipóteses, com a aplicação da modelagem de equações estruturais, evidenciam que os elementos do marketing mix relacionamse de maneira diferenciada com as dimensões que antecedem o brand equity e que apenas a dimensão lealdade é significativa na formação das marcas de lojas.

Palavras-chave: Brand Equit. Marketing Mix. Marcas de Lojas.

\section{Abstract}

This article evaluates the relationships between the elements of the marketing mix and the creation of brand equity (BE), following a seminal conceptual framework in

1 Doutorado em Gestão pela Faculdade de Economia da Universidade de Coimbra (FEUC) na especialidade em Marketing. Professor assistente 4 da Universidade de Fortaleza (UNIFOR). E-mail: sarto@unifor.br. Endereço profissional: Universidade de Fortaleza, Av. Washington Soares, 1321. Fortaleza-CE - Brasil. Telefone: (85) 3477-3192.

2 Professor doutor da Faculdade de Economia da Universidade de Coimbra - Programa de Doutoramento em Gestão. E-mail: acoelho@fe.uc.pt. Endereço profissional: Av. Dias da Silva, 165. Coimbra - Portugal.

3 Doutorado em Economia Industrial e da Tecnologia - University of Reading (UR), Inglaterra. Pós-doutor pela University of Sussex - UK, pós-doutor pela Universidade Federal do Rio Grande do Sul (UFRGS) e professor do PPGA da Universidade de Fortaleza (UNIFOR). E-mail: ednilson@unifor.br. Endereço profissional: Universidade de Fortaleza, Av. Washington Soares, 1321. Fortaleza-CE - Brasil. Telefone: (85) 3477-3229. 
the marketing literature. The study is based on individual perceptions of 571 selected customers. These perceptions were collected in the context of an orientated top of mind comprising eight brands stores in Fortaleza. The results of the hypotheses tests, with the application of structural equation modeling, show that the elements of the marketing mix relate differently to the dimensions prior to the $B E$ and that only the loyalty dimension is significant in the formation of stores brands.

Keywords: Brand Equity. Marketing Mix. Stores'Brands.

\section{Introdução}

O tema deste estudo trata da identificação e avaliação do impacto dos antecedentes do brand equity no contexto das marcas de vestuários de lojas na cidade de Fortaleza. Entre esses antecedentes, encontramse os esforços de marketing da empresa, materializados no seu mix de marketing, mas também variáveis que derivam do comportamento pessoal relativo às marcas de lojas de vestuário.

Dentre a pletora de estudos relacionados com o marketing nos últimos anos, nas principais revistas acadêmicas, dois temas têm sido explorados, debatidos e reputados como relevantes no contexto prático e teórico, quais sejam: as marcas próprias (de lojas) e o brand equity. Embora, em sua maioria, os estudos sobre esses temas tenham ocorrido de maneira isolada, eles se sobrepõem parcialmente entre si.

Apesar desse nível atual de atenção aos temas, o interesse pelo estudo das marcas próprias por parte dos acadêmicos e praticantes do marketing no mundo não é novidade, pois, já em meados do século passado, alguns pesquisadores já apresentavam estudos empíricos em revistas de referência, como Cunninghan (1961), Frank e Boyd (1965), Myers (1967) e, mais recentes, neste século, D'Astou e SaintLouis (2005), Hansen, Singh e Chintagunta (2006), Ailawdi, Pauwels e Steenkamp (2008) e Liljander, Polsa e Van Riel (2009), dentre outros.

A maior notoriedade e interesses foram desencadeados a partir do trabalho de Quelch e Harding (1996), que advertiram o mundo acadêmico e empresarial para a batalha entre as marcas próprias e de fabricantes. O artigo destaca que, à época, as marcas próprias nos Estados Unidos 
detinham fatias de mercados individuais maiores do que as marcas nacionais mais fortes em 77 dentre as 250 categorias de produtos de supermercados, e, no todo, ocupavam o segundo ou terceiro lugar em 100 dessas categorias.

O relatório publicado pelo AC Nielsen, que trata do poder das marcas próprias em 2005, faz uma revisão do crescimento e das tendências dessas marcas. Ele mostra a presença delas em 38 países do mundo, em 80 categorias diferentes de produtos, tendo uma participação global de $17 \%$ no mundo, 23\% na Europa, 16\% na América do Norte, 6\% nos mercados emergentes, $4 \%$ na Ásia e 2\% na América Latina. Nos mercados emergentes, a taxa de crescimento das marcas próprias é da ordem de $11 \%, 7 \%$ na América do Norte, 5\% na América Latina e Ásia e 4\% na Europa, para um crescimento global de 5\%. Em termos de preços praticados nos 38 países, 80 categorias dos produtos de marcas próprias apresentam, em média, preços 31\% abaixo dos preços de produtos similares de marcas tradicionais. A variação dos preços por regiões foi desde um diferencial de $50 \%$ abaixo na Polônia até apenas $10 \%$ em Hong Kong.

Essa realidade, ou seja, as evidências de que as marcas próprias estão presentes no mundo em várias categorias de produtos, com preços na maior parte inferiores, mas com qualidade aparentemente idêntica às marcas de fabricantes, estimula a formulação dos seguintes questionamentos: quais são os elementos do marketing mix que impulsionam as dimensões do brand equity das marcas de lojas de vestuários? Quais as dimensões que formam o brand equity das marcas de lojas? Quais são as características individuais do consumidor e a personalidade das marcas das lojas?

Este artigo tem como objetivo central identificar os elementos do marketing mix que impulsionam e formam as dimensões do brand equity das marcas de lojas de vestuário, e, de maneira secundária, caracterizar o consumidor e identificar a personalidade das marcas de lojas.

Além desta introdução, o artigo está estruturado da seguinte forma: a seção 1 apresenta a revisão da literatura e explicita as hipóteses do 
estudo. Na sequência, a seção 2 apresenta a metodologia da pesquisa, contemplando a indicação da população e amostra, tipologia, técnica analítica e modelo estrutural. A seção 3 apresenta a análise e discussão dos resultados a partir do suporte ou não às hipóteses testadas. Finalizando o artigo, as considerações finais são apresentadas na seção 4; e as limitações do estudo, na seção 5.

\section{Revisão da literatura e hipóteses}

Brand equity refere-se à obtenção de valor superior por uma marca comparado àquele que se obteria se o produto ou serviço não fosse identificado por aquela marca (AAKER, 1991; KELLER, 1993; AAKER; BREL, 1993). Nesse sentido, Yoo, Donthu e Lee (2000), a partir do modelo de Aaker (1991), desenvolveram um quadro conceitual de brand equity (Figura 1). Originalmente, Aaker (1991) sugeriu que, em primeiro lugar, o brand equity gera valor tanto para a empresa como para o cliente. Num primeiro momento, a empresa garante uma certa proposta de valor ao cliente. Num segundo momento, a satisfação e a lealdade do cliente produzem valor para a marca e para a empresa.

Yoo, Donthu e Lee (2000) expandiram o modelo de Aaker (1991) de duas maneiras. Na primeira, eles separaram o conceito de brand equity, colocando-o entre as "dimensões de brand equity" e os "valores gerados para os clientes e para a empresa". Essa separação mostra que o conceito de brand equity relaciona-se individualmente com suas dimensões. Esse modelo mostra como as dimensões do brand equity contribuem para o valor da empresa. Adicionalmente, Yoo, Donthu e Lee (2000) inseriram como antecedentes dessas dimensões as atividades de marketing, que, na opinião deles, têm significativos efeitos nas dimensões do brand equity.

Os elementos selecionados do programa de marketing são os preços, a imagem da loja, a intensidade de distribuição, as despesas com propaganda e as promoções de preços ou negociações. Embora essas variáveis não atendam ao domínio completo do marketing, representam ações típicas da estratégia da empresa, com grande impacto potencial 
na imagem da marca. Sabendo que essas atividades de marketing contribuem para o brand equity, a sua gestão, de forma criativa e apurada, possibilita o desenvolvimento de planos de marketing mais eficazes. Os gestores de marketing devem, então, promover atividades que ajudem a construir e solidificar o brand equity, por meio de uma gestão consequente dos planos de marketing da empresa.

Figura 1 - Quadro conceitual do brand equity

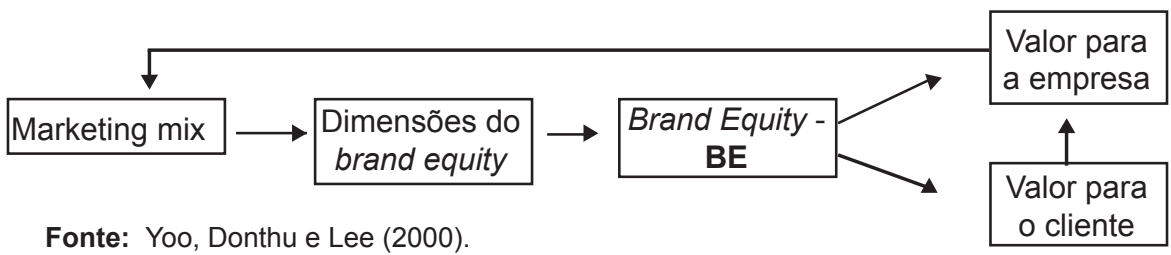

Yoo, Donthu e Lee (2000) definem brand equity como a diferença na escolha por parte do consumidor entre um produto com marca e um produto sem marca, tendo em conta um mesmo nível de características. Essa definição lida com a comparação de dois produtos idênticos em todos os aspectos, exceto na marca (por exemplo, um produto Lacoste comparado com um produto sem marca). Todos os consumidores têm uma impressão do que a Lacoste transmite sobre um produto, que é diferente da impressão transmitida por um produto sem nome. O brand equity da marca Lacoste é o valor extra incorporado em seu nome, como é percebido pelo consumidor, em comparação com um produto idêntico, mas sem nome. A diferença na escolha do consumidor sobre esses dois produtos pode ser avaliada medindo-se a intenção de comprar ou a preferência pela marca em comparação com a contrapartida de um produto sem nome.

De acordo com Aaker (1991), brand equity é um conceito multidimensional. Consiste na qualidade percebida, no conhecimento sobre a marca, nas associações à marca e em outros ativos relacionados com ela. Outros pesquisadores identificaram dimensões semelhantes. Shocker e Weitz (1988) propuseram a lealdade à marca e as associações a ela; Keller (1993) sugere o conhecimento da marca, compreendendo 
a sua consciência e imagem. Tendo em conta os diversos estudos, Yoo, Donthu e Lee (2000) reconhecem que a lealdade à marca, a qualidade percebida, o conhecimento dela e as associações positivas são as dimensões mais comuns do brand equity.

Para Yoo, Donthu e Lee (2000), um brand equity alto implica que os clientes têm fortes e positivas associações relacionadas com a marca; percebem a marca como de alta qualidade, e, por conseguinte, são leais a ela. Nesse contexto, o modelo apresentado na Figura 1 transmite a ideia de que as dimensões do brand equity o aumentam, porque cada uma delas está relacionada positivamente com ele.

Qualquer esforço de marketing será positivamente relacionado com o brand equity quando se leva a uma resposta comportamental mais favorável ao produto. Tal como proposto no âmbito conceitual, os esforços gerenciais manifestam-se em ações de marketing controladas e relacionadas com a imagem da marca, através da mediação das dimensões do brand equity. Portanto, para criar, gerenciar e explorar o brand equity, os impactos dos esforços de marketing nas dimensões do brand equity devem ser determinados (YOO; DONTHU ; LEE, 2000).

\section{Preço}

As marcas de preços altos são geralmente consideradas como sendo de maior qualidade e menos vulneráveis aos preço,s podendo competir com vantagem com as marcas de preço baixo (OLSON, 1977; KAMAKURA ; RUSSELL, 1993; ANSELMSSON, JOHANSSON ; PERSSON, 2007; CHEN ; GREEN, 2009). Rao e Monroe (1989) afirmam que há uma relação positiva entre preço e qualidade percebida. Ao aumentar a qualidade percebida, relaciona-se o preço positivamente às dimensões do brand equity (YOO; DONTHU ; LEE, 2000).

Consumidores leais à marca estão dispostos a pagar o preço total por ela, porque são menos sensíveis ao preço do que os consumidores não leais. Assim, a alteração do nível de preços não afeta a lealdade à marca. Yoo, Donthu e Lee (2000) também afirmam que não há nenhum 
relacionamento direcional entre o preço e as associações à marca, porque os preços baixos ou altos podem ser fortemente ligados aos benefícios que cada marca traz à memória dos consumidores. Portanto, propõem-se as seguintes hipóteses:

- Ha1 - Há uma relação positiva entre os preços altos das marcas das lojas e a qualidade percebida destas marcas.

\section{Imagem das lojas}

Uma gestão cuidadosa do canal de distribuição entre as ferramentas de marketing contribui para incrementar o brand equity (SRIVASTAVA; SHOCKER, 1991). Em um canal de distribuição, varejistas encontram os consumidores finais de uma empresa. Em particular, a distribuição através das lojas de boa imagem são indicadores da qualidade. Snipes, Thomson e Oswald (2006) e Chen e Green (2009) encontram efeitos positivos significativos da imagem da loja na qualidade percebida. $O$ nome da loja é uma pista vital extrínseca da qualidade percebida. Ela é percebida de maneira diferente, dependendo do que oferece o varejista.

A boa imagem da loja atrai mais atenção, contatos e visitas de cliente potenciais. Além disso, tais lojas proporcionam uma maior satisfação ao consumidor e estimulam um boca a boca positivo entre os consumidores (ZEITHAML, 1988; RAO e MONROE, 1989). Portanto, a distribuição de uma marca através de lojas com uma boa imagem vai criar associações de marca mais positivas (YOO, DONTHU, LEE, 2000). Liljander, Polsa e Van Riel (2009) afirmam que a imagem das mercadorias (camisas masculinas) e a qualidade dos serviços ofertados nas lojas são refletidos na imagem das lojas de vestuário. Diante dessa sustentação, propõem-se as seguintes hipóteses:

- Hb1 - Há uma relação positiva entre a imagem da loja das marcas das lojas e a qualidade percebida dessas marcas.

- Hb2 - Há uma relação positiva entre a imagem da loja das marcas das lojas e as associações e conhecimento dessas marcas. 


\section{Intensidade da distribuição}

A distribuição é dita como intensiva quando os produtos são colocados em um grande número de lojas para cobrir o mercado. Os varejistas, para melhorar a imagem de um produto e obter apoio substancial, preferem uma distribuição mais exclusiva ou seletiva, e não de forma intensiva. Os consumidores estarão mais satisfeitos, no entanto, quando um produto estiver disponível em um número maior de lojas, pois aumenta a acessibilidade dos produtos (FERRIS; OLIVER ; KLUYVER, 1989; SMITH, 1992; CHEN ; GREEN, 2009). A distribuição intensiva reduz o tempo na procura das lojas, proporcionando comodidade na compra e tornando mais fácil obter os serviços ou produtos. Conforme aumenta a intensidade da distribuição, os consumidores têm mais tempo e percebem mais valor nos produtos. Esse maior valor leva a uma maior satisfação dos consumidores, a uma maior qualidade percebida, a uma maior lealdade à marca, a associações mais positivas e, consequentemente, a um maior brand equity (YOO; DONTHU ; LEE, 2000). Assim, foram propostas as hipóteses a seguir:

- Hc1 - Há uma relação positiva entre a intensidade de distribuição das lojas e a qualidade percebida dessas marcas.

- Hc2 - Há uma relação positiva entre a intensidade de distribuição das marcas das lojas e a lealdade dessas marcas.

- Hc3 - Há uma relação positiva entre a intensidade de distribuição das lojas e associações e conhecimento dessas marcas.

\section{Gastos com propaganda}

Altos gastos com propaganda mostram que a empresa está investindo na marca, o que se traduz em uma qualidade superior (KIRMANI ; WRIGHT, 1989). Além disso, Archibald, Haulman e Moody (1983) encontraram que os níveis de gastos com propaganda são bons indicadores não apenas de elevada qualidade, mas também de uma boa compra. Aaker e Jacobson (1994), Belch e Belch (2007) e Chen e Green (2009) também encontraram uma relação positiva entre a 
propaganda e a qualidade percebida. Assim, gastos com propaganda estão positivamente relacionados com a qualidade percebida e ainda incrementam o brand equity.

Para Yoo, Donthu e Lee (2000), a propaganda desempenha um papel fundamental no aumento do conhecimento da marca, bem como na criação de fortes associações a ela. Esses conjuntos de assertivas levaram às hipóteses a seguir:

- Hd1 - Há uma relação positiva entre os gastos com propaganda das marcas das lojas e a qualidade percebida dessas marcas.

- Hd2 - Há uma relação positiva entre os gastos com propaganda das marcas das lojas e a lealdade dessas marcas.

- Hd3 - Há uma relação positiva entre os gastos com propaganda das lojas das marcas de lojas e associações e conhecimento delas.

\section{Preços negociados}

A promoção de vendas, em especial, as promoções de preços ou preços negociados (por exemplo, a redução de preços a curto prazo, vendas especiais, cupons distribuídos, ofertas, descontos e restituições), corroem o brand equity ao longo do tempo, apesar do imediato ganho financeiro a curto prazo. A promoção de vendas não pode ser uma forma conveniente para construir brand equity, pois é facilmente copiado e o desempenho a longo prazo fica neutralizado (AAKER, 1991).

Yoo, Donthu e Lee (2000) entendem que as promoções de preço parecem não estar relacionada com a lealdade à marca, embora sejam encontradas de forma consistente para forçar a troca temporária de marca. As promoções, muitas vezes, não conseguem estabelecer um padrão de compra após um julgamento inicial por parte do consumidor. Isso acontece porque os consumidores estão momentaneamente atraídos para a marca pelo utilitarismo da transação, que resulta das promoções de preços, mas, quando terminar a promoção, eles perdem o interesse pela marca. Assim, a mudança de marca depois do fim das 
promoções pode não ocorrer, a menos que a marca promovida seja percebida como superior e satisfaça as necessidades dos consumidores. Apoiando-se mais uma vez em Yoo, Donthu e Lee (2000), elaboraram-se as seguintes hipóteses:

- He1 - Há uma relação negativa entre os preços negociados das lojas das marcas de lojas e a qualidade percebida destas.

- He2 - Há uma relação negativa entre os preços negociados das lojas das marcas de lojas e as associações e conhecimento destas.

\section{As dimensões do brand equity}

A compreensão correta do fenômeno de brand equity passa também pela compreensão das suas dimensões (AAKER, 1991) e da influência delas na determinação do brand equity.

Yoo, Donthu e Lee (2000) apoiam-se em Zeithaml (1988), que define a qualidade percebida como um julgamento (subjetivo) sobre a excelência global de um produto ou a sua superioridade atribuída pelo consumidor. A experiência pessoal com produtos, as necessidades específicas e as situações de consumo podem influenciar no julgamento subjetivo do consumidor sobre a qualidade. A alta qualidade percebida significa que, através da experiência de longo prazo com a marca, os consumidores reconhecem uma diferenciação e superioridade desta. Nessa linha de pensamento, a qualidade percebida é identificada como um componente do valor da marca e, portanto, a alta qualidade percebida conduz o consumidor a escolher uma marca, em vez de outras. Portanto, o grau de qualidade com que a marca é percebida pelos consumidores contribui positivamente para o brand equity (CHEN ; GREEN, 2009).

Oliver (1997) define a lealdade à marca como um compromisso profundamente arraigado de repetir a compra de um produto ou serviço preferido, consistentemente, ao longo do tempo, apesar de influências situacionais e de esforços de marketing para mudar o comportamento do consumidor. A lealdade à marca faz com que o consumidor compre 
uma marca de maneira rotineira e seja resistente à mudança para outra marca. Assim, na medida em que os consumidores são leais à marca, proporcionam um aumento do brand equity (DATTA, 2003).

A imagem da marca está associada a um forte conhecimento dela. Aaker (1991,) define associações à marca como qualquer coisa ligada na memória a uma marca e a imagem da marca como um conjunto de associações de alguma forma significativas a uma marca. Associações à marca são complexas, ligadas às outras, e consistem em várias ideias, episódios, ocorrências e fatos que estabelecem uma rede sólida de conhecimento da marca. As associações são mais fortes quando baseadas em muitas experiências ou exposições nas mídias (ALBA ; HUTCHINSON, 1987; AAKER, 1991). Associações à marca que resultam em alta percepção são positivamente relacionados com o brand equity. Elas podem ser um sinal de qualidade e compromisso e ajudam um comprador a considerar a marca no ponto de compra, levando a um comportamento favorável para a marca (WALSH ; MITCHELL, 2005). Essas três dimensões, apoiadas nos estudos de Yoo, Donthu e Lee (2000), respaldados por Aaker (1991), conduzem às seguintes hipóteses:

- Hf1 - Há uma relação positiva entre a qualidade percebida da marca da loja e o brand equity da loja.

- Hg1 - Há uma relação positiva entre a lealdade da marca da loja e o brand equity da loja.

- Hh1 - Há uma relação positiva entre as associações e conhecimento da marca da loja e o brand equity da loja.

\section{As características do consumidor}

Neste artigo, propõe-se uma ampliação do modelo sugerido por Yoo, Donthu e Lee (2000), com a inclusão das "variáveis individuais", tendo em vista as proposições teóricas de que elas apresentam-se relacionadas com o brand equity. Essa inserção deve-se à importância dessas variáveis para as estratégias de marketing (TYNAN; DRAYTON, 1987) e pela relação destas com o setor estudado (vestuário). Nesse 
sentido, Gutman e Mills (1982) referem-se especificamente a estilo de vida, tal como atitudes, interesses, opiniões e comportamentos dos consumidores, e como eles se relacionam com a aquisição de mercadorias de moda.

O termo "moda", em um sentido amplo, é frequentemente usado para explicar as tendências no consumo de bens (BAKEWELL et al., 2006). A noção de moda também envolve o comportamento do consumo que envolve gostos e valores individuais, pois os estilos que estão na moda são geralmente aceitos por um grande grupo de pessoas em um determinado momento, significando uma identidade distintiva no meio social (GRONOW, 1997). Isso ocorre quando o termo se refere a vestuário e a outros objetos materiais usados por pessoas (KAISER, 1997). Nesse caso, assume-se a perspectiva apresentada por Schindler e Holbrook (1993): a moda abraça todos os componentes mencionados, incluindo toda a gama de elementos visuais que podem levar à percepção da moda (por exemplo, corte de cabelo, roupas, joias e acessórios). Assim, o termo "moda" pode ser aplicado a todos os aspectos da aparência pessoal de alguém que forneça valor utilitarista e hedonista para o consumidor (BANNISTER ; HOGG, 2004). Nesse sentido, elegeu-se a dimensão "orientação para a moda", construto desenvolvido por Gutman e Mills (1982), composto das variáveis "líder em moda (LDM)" e "bem vestido (BV)" para desenvolverem as hipóteses a seguir:

- Hi1 - Há uma relação positiva entre o construto "LDM" e o brand equity da loja.

- Hk1 - Há uma relação positiva entre o construto "BV" e o brand equity da loja.

Seis critérios são frequentemente sugeridos na literatura de marketing para avaliar a atratividade dos segmentos de mercado (FRANK et al., 1972; WEDEL ; KAMAKURA, 2000; BAKER, 2003; KOTLER ; KELLER, 2006): a identificabilidade, a substanciabilidade, a acessibilidade, a capacidade de resposta, a estabilidade e a acionabilidade - ou seja, são passíveis de serem atingíveis se a 
sua identificação fornece uma orientação para as decisões sobre a especificação efetiva dos instrumentos de marketing a serem usados.

Nesse contexto, selecionou-se a variável "planejador (PLN)", desenvolvida por Gutman e Mills (1982), para definir grupos de consumidores com características voltadas para as compras, ou seja, utilizou-se do critério para avaliar a atratividade de mercado por meio da identificação de clientes. Portanto, formulou-se a seguinte hipótese:

- Hq1 - Há uma relação positiva entre o construto "PLN" e o brand equity da loja.

Os estudos de Tynan e Drayton (1987) demonstram resultados significativos e não significativos quando se relacionam os traços de personalidade e categorias de produtos, devendo-se ressaltar que os traços de personalidade, as categorias de produto, bem como as metodologias aplicadas eram distintas entre si. De maneira contrária, Buil, De Chernatony e Martínez (2008) não encontraram relação entre os traços de personalidade e a empresa.

Para ultrapassar essas dificuldades, decidiu-se abdicar dos traços de personalidade individual e dar preferência aos traços de personalidade da marca, que poderiam ser um melhor preditor. Nesse contexto, relacionou-se a hipótese Hs1 entre os construtos brand equity e personalidade da marca. Para esse propósito, adotou-se o conceito de "personalidade da marca", desenvolvido por Aaker (1997, p. 58), tido como "um conjunto de características humanas que se associam a uma marca", portanto:

- Hs1 - Há uma relação positiva entre o construto personalidade da marca da loja (PSML) e o brand equity da loja.

As hipóteses formuladas são graficamente representadas na Figura 2. 
Figura 2 - Modelo conceitual da pesquisa

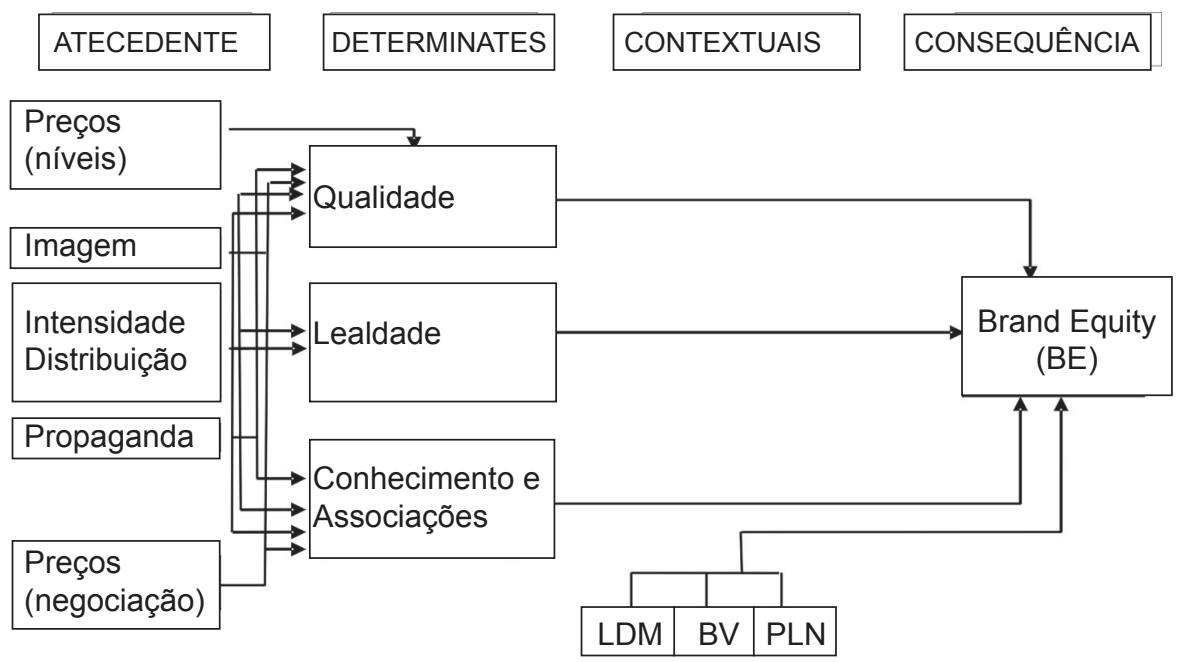

\section{Quadro metodológico da pesquisa}

Foi definida como população-alvo os frequentadores de três shoppings, quatro repartições públicas, três universidades e uma faculdade da cidade de Fortaleza, cujas identidades não foram reveladas. A amostra deste estudo é caracterizada como sendo de natureza não probabilística por conveniência, devido às limitações e dificuldades de recursos para se garantir a aplicação da técnica de amostragem probabilística (MALHOTRA, 2006).

Para coleta dos dados, utilizaram-se questionários estruturados não disfarçados, sempre com as mesmas perguntas e opções de resposta (MATTAR, 2001). Para a operacionalização da aplicação dos questionários, foram utilizados quatro estudantes bolsistas dos programas de pesquisa da Universidade de Fortaleza e três professores voluntários de três faculdades de Fortaleza. Devidamente identificados, apresentaram, aplicaram e recolheram os questionários pessoalmente, sem interferência oral, deixando os 610 voluntários à vontade para responderem a pesquisa. Um total de 571 questionários foram validados, já que 39 foram eliminados por imperfeições de preenchimento. Em 
termos de tamanho, salienta-se que a amostra cumpriu as exigências mínimas de pelo menos cinco respondentes para cada parâmetro estimado (HAIR et al., 2005). Entre os respondentes, 54,6\% eram do sexo feminino, 43\% tinham a idade entre 15 e 24 anos, $61 \%$ tinham o segundo grau completo e o terceiro grau incompleto, 69,9\% detinham uma renda familiar de 3 a 20 salários mínimos. 73\% da área geográfica de Fortaleza foram contempladas por moradores dos 114 bairros, sendo que, destes, 68,48\% haviam comprado vestuários com as marcas de marcas de lojas.

Os construtos do marketing mix preços (PML), imagem da loja (IML), intensidade de distribuição (IDML), gastos com propaganda (GPML) e preços negociados (PNML); e os construtos brand equity (BEML) e qualidade percebida (QPML), lealdade ( $L M L)$ e conhecimento e associações (CAML) foram mensurados com o uso da escala Likert de cinco pontos, conforme os indicadores desenvolvidos por Yoo, Donthu e Lee (2000). Já o construto "orientação para a moda e para as compras" baseou-se no estudo de Gutman e Mills (1982). Finalmente, a "personalidade da marca" foi definida a partir de Aaker (1997).

No teste das hipóteses do modelo proposto, utilizou-se a modelagem de equações estruturais (MEE) que constitui uma técnica multivariada que combina a análise fatorial exploratória com a estimação de um conjunto de equações simultâneas. A MEE foi considerada a técnica analítica apropriada e mais eficiente para esta pesquisa, porque o modelo apresenta um conjunto de equações de regressão múltiplas separadas, mas interdependentes, que devem ser estimadas simultaneamente (HAIR et al., 2005).

A análise foi iniciada com o modelo de mensuração, por meio da aplicação da análise fatorial confirmatória (AFC) para verificar as propriedades psicométricas das escalas e testar as relações preestabelecidas (HAIR et al., 2005). Os índices de ajuste estão dentro dos valores considerados satisfatórios (Tabela 1). 
Tabela 1 - Medidas de ajuste do modelo de mensuração das marcas de lojas

\begin{tabular}{l|c|cc|cc}
\hline Medidas & $\begin{array}{c}\text { Ajuste } \\
\text { absoluto }\end{array}$ & \multicolumn{2}{|c|}{ Ajuste incremental } & \multicolumn{2}{|c}{$\begin{array}{c}\text { Ajuste } \\
\text { parcimonioso }\end{array}$} \\
\hline Qui-quadrado $\left(\mathrm{X}^{2}\right)$ & 1148,407 & AGFI & 0,880 & PNFI & 0,775 \\
Graus de liberdade & 624,000 & TLI ou NNFI & 0,955 & PGFI & 0,723 \\
CMIN/DF & 1,840 & NFI & 0,921 & AIC & 1460,407 \\
Nível de significância $(p$ & 0,000 & CFI & 0,962 & & \\
value) & 0,904 & & & & \\
GFI & 0,038 & & & & \\
RMSEA & & & & & \\
\hline
\end{tabular}

Fonte: Pesquisa de campo.

Hair et al. (2005) recomendam que logo que o modelo de ajuste geral tenha sido analisado, a mensuração de cada construto pode ser avaliada quanto à unidimensionalidade e confiabilidade. Assim, procedeuse a validade discriminante dos construtos que integram o modelo final. Verificou-se que o quadro da correlação entre cada par de construtos é inferior à variância extraída de cada um deles, obtendo-se resultados compatíveis com os aceitos na literatura (Variância Extraída superior a 0,5 e Confiabilidade Composta excede a 0,7), conforme Tabela 2.

Tabela 2 - Validade discriminante dos construtos das marcas das lojas

\begin{tabular}{|c|c|c|c|c|c|c|c|c|c|c|c|c|c|c|c|c|}
\hline & DP & PML & IML & IDML & GPML & PNML & BEML & CAML & LML & QPML & PSML & LDM & BV & PLN & $\mathrm{CC}$ & VE \\
\hline PML & 0,721 & 0,750 & & & & & & & & & & & & & 0,797 & 0,675 \\
\hline IML & 0,939 & 0,405 & 0,863 & & & & & & & & & & & & 0,863 & 0,760 \\
\hline IDML & 0,919 & 0,185 & 0,153 & 0,834 & & & & & & & & & & & 0,837 & 0,719 \\
\hline GPML & 0,921 & 0,044 & 0,050 & 0,082 & 0,875 & & & & & & & & & & 0,877 & 0,705 \\
\hline PNML & 1,055 & $-0,059$ & $-0,075$ & 0,273 & 0,197 & 0,867 & & & & & & & & & 0,871 & 0,694 \\
\hline BEML & 1,062 & 0,046 & 0,188 & 0,036 & 0,100 & 0,135 & 0,930 & & & & & & & & 0,931 & 0,771 \\
\hline CAML & 0,987 & 0,013 & 0,157 & 0,077 & 0,193 & 0,163 & 0,193 & 0,939 & & & & & & & 0,940 & 0,757 \\
\hline LML & 1,028 & 0,117 & 0,209 & 0,014 & 0,107 & 0,106 & 0,369 & 0,316 & 0,902 & & & & & & 0,902 & 0,754 \\
\hline QPML & 0,789 & 0,335 & 0,648 & 0,188 & 0,134 & $-0,012$ & 0,215 & 0,255 & 0,179 & 0,936 & & & & & 0,937 & 0,716 \\
\hline PSML & 0,792 & 0,323 & 0,544 & 0,177 & 0,320 & 0,139 & 0,303 & 0,387 & 0,304 & 0,668 & 0,648 & & & & 0,612 & 0,442 \\
\hline LDM & 1,085 & 0,058 & 0,119 & 0,120 & 0,102 & 0,073 & 0,159 & 0,179 & 0,168 & 0,104 & 0,264 & 0,679 & & & 0,708 & 0,468 \\
\hline BV & 0,777 & $-0,020$ & 0,116 & 0,079 & $-0,052$ & $-0,075$ & 0,033 & 0,109 & $-0,099$ & 0,215 & 0,207 & 0,372 & 0,630 & & 0,643 & 0,473 \\
\hline PLN & 1,249 & 0,012 & 0,043 & 0,097 & 0,057 & 0,072 & 0,091 & 0,129 & 0,126 & 0,026 & 0,255 & 0,294 & 0,193 & 0,755 & 0,784 & 0,654 \\
\hline
\end{tabular}

Fonte: Pesquisa de campo. Nota: A diagonal principal apresenta o alpha de Cronbach; DP = Desvio Padrão; CC=Confiabilidade Composta; VE = Variância Extraída. 
A partir dos resultados, procedeu-se ao exame do desempenho geral do modelo estrutural das marcas de lojas. As medidas absolutas de ajustamento (apontam o grau em que o modelo prediz a matriz de covariância ou de correlação observada) foram adequadas, com os índices em níveis de aceitação periférica, uma vez que não há referência estabelecida (HAIR et al., 2005). Todavia, Hair et al.(2005) afirmam que a medida aplicável para avaliar um único modelo é a medida de $\mathrm{X}^{2}$ normado com limite superior a 5 . Portanto, os três tipos de medidas gerais de ajuste observados revelam um padrão consistente de apoio para o modelo das marcas de lojas (Tabela 3).

Tabela 3 - Medidas de ajuste do modelo estrutural das marcas de lojas

\begin{tabular}{|c|c|c|c|c|c|}
\hline Medidas & $\begin{array}{l}\text { Ajuste } \\
\text { absoluto }\end{array}$ & \multicolumn{2}{|c|}{$\begin{array}{c}\text { Ajuste } \\
\text { incremental }\end{array}$} & \multicolumn{2}{|c|}{$\begin{array}{c}\text { Ajuste } \\
\text { parcimonioso }\end{array}$} \\
\hline Qui-quadrado $\left(\mathrm{X}^{2}\right)$ & 1415,776 & \multirow{7}{*}{$\begin{array}{c}\text { AGFI } \\
\text { TLI ou } \\
\text { NNFI } \\
\text { NFI } \\
\text { CFI }\end{array}$} & 0,866 & PNFI & 0,794 \\
\hline Graus de Liberdade & 652,000 & & 0,937 & PGFI & 0,742 \\
\hline CMIN/DF & 2,171 & & 0,902 & \multirow[t]{5}{*}{ AIC } & \multirow[t]{5}{*}{1671,776} \\
\hline $\begin{array}{l}\text { Nível de significância ( } p \\
\text { value) }\end{array}$ & 0,000 & & 0,944 & & \\
\hline GFI & 0,888 & & & & \\
\hline RMSEA & 0,045 & & & & \\
\hline RMR ou SRMR & 0,106 & & & & \\
\hline
\end{tabular}

Fonte: Pesquisa de campo.

\section{Análise e discussão de resultados}

A Tabela 4 resume os resultados do modelo estrutural que respondem ao conjunto de hipóteses testadas neste estudo.

A hipótese Ha1 - de que há uma relação positiva entre preços altos das marcas de lojas e a qualidade percebida dessas marcas - não foi suportada no contexto das marcas de vestuário de lojas, embora a rejeição tenha se dado a um nível de significância muito próximo a t=1,65. Possivelmente a rejeição dessa hipótese está relacionada a uma menor precisão na concepção dos conceitos de preço e de qualidade, uma vez que esses conceitos são ambíguos, isto é, difíceis de serem assimilados 
de uma maneira uníssona por parte do consumidor (SWINKER e HINES, 2006). Adicionalmente, o consumidor em geral tem percepções em relação a preço como um sacrifício para obtenção de um produto e, dessa maneira, confunde facilmente adjetivos imprecisos relacionados à qualidade que é um conceito multidimensional (YOO, DONTHU e LEE, 2000).

As hipóteses suportadas ( $\mathrm{Hb} 1 \mathrm{e} \mathrm{Hb} 2)$ relacionam o construto imagem com a qualidade percebida e com o conhecimento associado às marcas de vestuário. Essas relações positivas encontram respaldo em Paulins e Geistfeld (2003), uma vez que, independentemente da categoria das marcas, os gestores devem zelar pela imagem de suas lojas, pois se trata de um impulsionador diretamente ligado com as características do consumidor.

Por sua vez, as hipóteses $\mathrm{Hc1}$, Hc2, Hc3 - que avaliam a relação da intensidade de distribuição das marcas de lojas com a qualidade percebida, com a lealdade e com o conhecimento e associação dessas marcas - não foram suportadas. Da mesma forma, a hipótese He1, relacionando preços negociados a qualidade percebida, também não foi suportada. Esses resultados não são considerados surpreendentes, tendo em vista a natureza dos produtos (vestuário), que não se adequam à estratégia de vendas intensivas, uma vez que o consumidor de vestuário é discriminatório por natureza e o conceito de qualidade é ambíguo (SWINKER e HINES, 2006). Esse fato deve-se também à estratégia de diferenciação das marcas de lojas, que buscam se apresentar como exclusivas para seus clientes, por meio de uma mídia mais seletiva.

A hipótese He2, relacionando preços negociados e conhecimento, foi suportada. Assim, desde que os preços constituam uma prática realizada pelas marcas de lojas, principalmente quando da renovação de suas prateleiras com a chegada das novas coleções, elas não impactam na qualidade percebida, mas impactam o conhecimento e associações às marcas. De fato, muitos consumidores associam essas marcas a preços e vantagens especiais. 
Foi igualmente encontrado suporte para as hipóteses $\mathrm{Hd} 1, \mathrm{Hd} 2$ e Hd3, que introduzem os efeitos dos gastos com propaganda. De fato, tratando-se de marcas próprias de lojas, com uma distribuição limitada, os efeitos da propaganda podem ser decisivos para atribuir algum tipo de conhecimento e associações às marcas, uma ideia de qualidade e gerar alguma lealdade à marca. Esses resultados corroboram os pontos de vista de Aaker (1998) e D'Astous e Saint-Louis (2005).

Assim, as relações mais significativas entre antecedentes e as dimensões do brand equity derivam essencialmente da imagem das lojas e dos impactos da propaganda, e, de forma mais parcial, dos efeitos dos preços negociados (uma associação significativa e outra não significativa). Por sua vez, preços negociados e intensidade da distribuição não exercem os efeitos positivos hipotetizados.

As hipóteses relativas aos impactos no brand equity mostram suporte para os efeitos da lealdade $(\mathrm{Hg} 1)$. A aceitação da relação positiva do construto lealdade com o brand equity das marcas de lojas se deve à experiência (compram ou usam) com essas marcas (KOTLER e KELLER, 2006). As hipóteses Hf1 e Hh1 não foram suportadas significativamente, o que evidencia que o brand equity das marcas de lojas não tem origem nem na qualidade nem nos conhecimentos e associações à marca. Como o conceito de qualidade é tido como ambíguo no segmento de vestuário, pode ser essa a razão para que a qualidade, o conhecimento e as associações não se relacionem com o brand equity.

Finalmente, quando se relaciona o perfil dos consumidores com a avaliação que fazem das marcas, os resultados dividem-se. Assim, encontraram suporte as hipóteses Hi1e Hs1. Elas sugerem que os líderes em moda e construto personalidade das marcas de lojas relacionam-se positivamente com o brand equity das marcas de lojas. Os conceitos "líder em moda", sugerido por Gutman e Mills (1982), e "personalidade da marca”, sugerido por Aaker (1997), apresentaram relação significativa com brand equity, presumivelmente por serem vistos comerciais no segmento de vestuário com apelos congruentes com esses conceitos. 
Contrariamente, os conceitos de planejador (Hk1) e bem vestido (Hq1), propostos por Gutman e Mills (1982), não são suportados suas relações com o brand equity das marcas de lojas, presumivelmente por não fazerem parte da cultura do consumidor fortalezense.

Tabela 4 - Modelo estrutural das marcas das lojas

\begin{tabular}{|c|c|c|c|c|}
\hline Hipóteses & $\begin{array}{l}\text { Coeficiente } \\
\text { Padronizado }\end{array}$ & C.R. $(t)$ & $\mathbf{P}$ & Conclusão \\
\hline Ha1. $\mathrm{PML} \rightarrow$ QPML (+) & 0,064 & 1,57 & 0,117 & Não suportado \\
\hline $\mathrm{Hb} 1 . \quad \mathrm{IML} \rightarrow$ QPML $(+)$ & 0,510 & 11,14 & $* * *$ & Suportado \\
\hline Hb2. IML $\rightarrow$ CAML $(+)$ & 0,177 & 3,83 & $* * *$ & Suportado \\
\hline Hc1. IDML $\rightarrow$ QPML (+) & 0,064 & 1,52 & 0,129 & Não suportado \\
\hline Hc2. IDML $\rightarrow$ LML (+) & 0,018 & 0,37 & 0,711 & Não suportado \\
\hline Hc3. IDML $\rightarrow$ CAML (+) & 0,004 & 0,08 & 0,936 & Não suportado \\
\hline Hd1. $\quad$ GPML $\rightarrow$ QPML $(+)$ & 0,086 & 2,10 & 0,036 & Suportado \\
\hline Hd2. $\quad$ GPML $\rightarrow$ LML $(+)$ & 0,123 & 2,59 & 0,010 & Suportado \\
\hline Hd3. $\quad$ GPML $\rightarrow$ CAML $(+)$ & 0,145 & 3,03 & 0,002 & Suportado \\
\hline He1. PNML $\rightarrow$ QPML (-) & $-0,009$ & $-0,21$ & 0,836 & Não suportado \\
\hline He2. $\quad$ PNML $\rightarrow$ CAML (-) & 0,127 & 2,54 & 0,011 & Suportado \\
\hline Hf1. QPML $\rightarrow$ BEML $(+)$ & 0,094 & 1,50 & 0,134 & Não Suportado \\
\hline Hg1. LML $\rightarrow$ BEML (+) & 0,301 & 6,54 & *** & Suportado \\
\hline Hh1. CAML $\rightarrow$ BEML $(+)$ & 0,043 & 0,92 & 0,358 & Não suportado \\
\hline Hi1. $\quad$ LDM $\rightarrow$ BEML (+) & 0,092 & 1,68 & 0,093 & Suportado \\
\hline Hk1. BV $\rightarrow$ BEML (+) & $-0,070$ & $-1,31$ & 0,190 & Não suportado \\
\hline Hq1. $\quad$ PLN $\rightarrow$ BEML $(+)$ & $-0,012$ & $-0,24$ & 0,811 & Não suportado \\
\hline Hs1. PSML $\rightarrow$ BEML (+) & 0,147 & 2,03 & 0,042 & Suportado \\
\hline
\end{tabular}

Fonte: Dados da pesquisa. Valor de $(t)$ usado 1,65 à nível de significância para $(p<0,05)$.

Essas evidências devem ser observadas por gestores e acadêmicos em estudos futuros e nas práticas de gestão corrente, pois os resultados apresentados mostram que os elementos do marketing mix, quando aplicados no contexto das marcas de lojas de vestuários, podem apresentar resultados e impactos diferenciados nos antecedentes do brand equity das marcas de lojas. Do mesmo modo, os resultados aqui encontrados são divergentes dos autores Yoo, Donthu e Lee (2000), que 
estudaram três categorias de produtos (aparelhos de TV, câmera de filmar e sapatos esportivos), nos quais apenas a intensidade de distribuição não é sustentada em relação ao conhecimento e às associações das marcas selecionadas no seu estudo. Ou seja, os resultados sobre a formação do brand equity têm que ser relativizados ao contexto e ao tipo de produtos de que falamos.

\section{Considerações finais}

As empresas têm dado atenção crescente à questão da marca como um fator de vantagem competitiva, podendo prognosticar resultados futuros. Significativa parte da literatura a respeito da gestão de marcas, a exemplo de Aaker (1991), Keller (1993) e De Chernatony e Mcdonald (2003), aborda estratégias para a criação de valor para a marca. Estudos sobre as medidas do brand equity baseado no cliente têm sido testados em diferentes contextos práticos e acadêmicos, verificando se essas estratégias estão sendo bem sucedidas ou não.

A utilização da escala multidimensional para a mensuração do brand equity tanto pode ser utilizada para estabelecer a posição relativa das marcas dentro do mercado como pode, através de uma série de medidas de uma marca específica, identificar como ela reage às ações de marketing executadas pela empresa ou pela concorrência. Além de uma avaliação geral do brand equity baseada no consumidor, a escala multidimensional proposta por Yoo e Donthu (2001) permite avaliar individualmente o desempenho da marca em cada uma das dimensões, o que pode servir para orientar de forma mais específica as ações necessárias para aumentar o valor do brand equity.

Ailawadi et al. (2003) e Atilgan et al.(2009) afirmam que é pouco provável, ou mesmo impossível, obter-se, por meio do brand equity baseado no consumidor, uma medida que satisfaça todas as características de uma marca, de maneira ideal, e que forneça indicações estimulantes para práticas dos gestores. Todavia, além das informações internas das empresas sobre o desempenho financeiro, os gestores 
podem beneficiar-se do monitoramento das variáveis do marketing mix (preço, imagem da loja, intensidade de distribuição, gastos com propaganda e preços promocionais). Elas antecedem e contribuem para a formação das dimensões do brand equity (qualidade percebida, lealdade à marca, conhecimento e associações à marca), bem como para o reforço e gerenciamento do brand equity das marcas de lojas de vestuário.

Acredita-se, portanto, que, ao adaptar as estratégias de marketing de acordo com as dimensões do brand equity, é possível projetar soluções para gerar valor ao consumidor de vestuário. Mais especificamente, os gerentes devem elaborar seus programas de marketing mix com vista a gerenciar e observar as relações causais com as dimensões do brand equity, a qualidade percebida, a lealdade à marca, o conhecimento e as associações às marcas de lojas.

Os resultados mostram bem as especificidades das marcas privadas ou de distribuidor. Mostram também como as suas dimensões impactam de uma forma moderada no brand equity dessas marcas. Isso significa, desde logo, dois desafios que ficam no ar: um para os profissionais de marketing, que podem aproveitar para adotar estratégias de marketing mais centradas e seletivas para aumentarem o seu brand equity; outro para os investigadores, para que se desenvolvam trabalhos que possam contribuir para uma redifinição das dimensões do brand equity para as marcas de distribuidor, que aqui aparecem limitadamente relacionadas com o brand equity global dessas marcas.

\subsection{Limitações da pesquisa}

Esta pesquisa traz algumas contribuições para as organizações em geral, especialmente para aquelas que atuam no segmento de vestuário. No entanto, ela tem também limitações, que podem ser exploradas em futuros estudos. Inicialmente, pode-se registrar o fato de o estudo ter se dado apenas na cidade de Fortaleza, com marcas privadas de lojas de vestuário. Futuras pesquisas, desenvolvidas em áreas mais vastas e 
com outros produtos com marca de distribuidor, podem ajudar a aferir a validade das conclusões aqui apresentadas.

Uma segunda limitação deste estudo refere-se ao uso de medidas de percepção a partir das métricas propostas por Yoo, Donthu e Lee (2000), e não a dados objetivos dos esforços de marketing das empresas. Seria significativo, a partir de uma perspectiva gerencial, utilizar dados de marketing a partir de fontes secundárias, tais como dados de scanner e relatórios de pesquisas publicadas ou dados das empresas detentoras das marcas submetidas ao teste de recall assistido deste estudo ou ainda de outras marcas não selecionadas para este estudo.

Por fim, usou-se um método de levantamento de campo baseado no questionário estruturado para testar as hipóteses de investigação. Esse método nem sempre é claro nas inferências e causalidades que permite estabelecer. Para investigar com mais rigor o impacto causal de cada esforço de marketing e das marcas de vestuário no processo de formação do brand equity, os investigadores poderiam projetar e realizar experiências observando o nível de esforço de marketing. Por isso, apela-se a futuras pesquisas a examinar o efeito das variáveis de marketing reais para determinar o brand equity das marcas de vestuário de lojas em um contexto longitudinal.

\section{Referências}

AAKER, D. A. Managing brand equity: Capitalizing on the value of a brand name. New York: Free Press, 1991.

AAKER, D. A. Marcas: brand equity gerenciando o valor da marca. São Paulo: Negócio, 1998.

AAKER, D. A.; BREL, A. C. Brand equity and advertising. Hillsdale, NJ: Lawrence Erlbaum.

AAKER, D. A.; JACOBSON, R. The financial information content of perceived quality. Journal of Marketing Research, v. 31, n. 5, p. 191201, 1994. 
AAKER, J. L. Dimensions of brand personality. Journal of Marketing Research, v. 34, n. 3, p. 347-356, 1997.

ACNIELSEN. The power of private label: a review of growth trends around the world. Executive News Report from ACNielsen Global Services, Sept 2005.

AILAWADI, K. L.; LEHMANN, D. R.; NESLIN, S. A. Revenue premium as an outcome measure of brand equity. Journal of Marketing, v. 67, n. 4, p. 1-17, 2003.

AILAWADI, K. L.; PAUWELS, K. E. M.; STEENKAMP, J. B. Privatelabel use and store loyalty. Journal of Marketing, v. 72, p. 19-30, 2008.

ALBA, J. W.; HUTCHINSON, J. W. Dimensions of consumer expertise. Journal of Consumer Research, v. 13, n. 4, p. 411-454, 1987.

ANSELMSSON, J.; JOHANSSON, U.; PERSSON, N. Understanding price premium for grocery products: A conceptual model of customerbased brand equity. Journal of Product at Brand Management, v. 16, n. 6, p. 401-414, 2007.

ARCHIBALD, R. B.; HAULMAN, C. A.; MOODY JR., C. E. Quality, price, advertising and published quality rating. Journal of Consumer Research, v. 9, n. 4, p. 347-356, 1983.

ATILGAN, E. et al. Customer-based brand equity for global brands: A multinational approach. Journal of Euromarketing, v. 18, p. 115-132, 2009.

BAKER, M. J. The marketing book. 5th ed. Oxford: ButterworthHeinemann, 2003.

BAKEWELL, C.; MITCHELL, V. W; ROTHWELL, M. U. K. Generation y fashion consciousness. Journal of Fashion Marketing and Management, v. 10, n. 2, p. 169-180, 2006.

BANNISTER, E. N.; HOGG, M. K. Negative symbolic consumption and consumers' drive for self-esteem: the case of the fashion industry. European Journal of Marketing. v. 8, n. 7, p. 850-868, 2004. 
BELCH, G. E.; BELCH, M. A. Advertising and promotion. Boston: McGraw-Hill Irwin, 2007.

BUIL, I; DE CHERNATONY, L; MARTINEZ, E. A cross-national validation of the consumer based brand equity scale. Journal of Product and Brand Management, v. 17, n. 6, p. 384-392, 2008.

CHEN, H.-C.; GREEN, R. D. Marketing mix and branding: Competitive hypermarket strategies. International Journal of Management and Marketing Research, v. 2, n. 1, p. 17-34, 2009.

CUNNINGHAM, R. M., Customer loyalty to store and brand. Harvard Business Review, v. 3, n. 9, p. 127-37, 1961.

D'ASTOUS, A; SAINT-LOUIS, O. National versus store brand effects on consumer evaluation of a garment. Journal of Fashion Marketing and Management, v. 9, n. 3, p. 306-317, 2005.

DATTA, P. R. The Determinants of brand loyalty. Journal of American Academy of Business, Cambridge, v.3, n. 1/2, p. 138-144, 2003.

DECHERNATONY, L.; MCDONALD, M. Creating powerful brands in consumer. service and industrial markets, Oxford: Elsevier, 2003.

FERRIS, P.; OLIVER, J.; KLUYVER, C. The relationship between distribution and market share. Marketing Science, v. 8, n. 2, p. 107127, 1989.

FRANK, R. E.; BOYD, H. W. Are The private-brand prone grocery customer really different? Journal of Advertising Research, v. 5, n. 4, p. 27-35, 1965.

FRANK, R. E.; MASSY, W. F.; WIND, Y. Market segmentation. Englewood Cliffs: Prentice-Hall, 1972.

GRONOW, J. The sociology of taste. London: Routledge, 1997.

GUTMAN, J.; MILLS, M. K. Fashion life style, self-concept, shopping orientation, and store patronage: an integrative analysis. Journal of Retailing, v. 58, n. 2, p. 64-86, 1982. 
HAIR JUNIOR, J. F. et al. Análise multivariada de dados. 5. ed. Porto Alegre: Bookman, 2005.

HANSEN, K.; SINGH, V; CHINTAGUNTA, P. Understanding storebrand purchase behavior Across Categories. Marketing Science, v. 25, n. 1, p. 75-90, 2006.

KAISER, S. B. The social psychology of clothing. 2nd ed. New York: Fairchild Publications, 1997.

KAMAKURA, W. A.; RUSSELL, G. J. Measuring brand value with scanner data. International Journal of Research in Marketing, v.10, p. 9-21, 1993.

KELLER, K. L. Conceptualizing, measuring, and managing customerbased brand equity. Journal of Marketing, v. 57, p. 1-22, 1993.

KIRMANI, A.; WRIGHT, P. Money talks: perceived advertising expenditures and expected product quality. Journal of Consumer Research, v. 16, p. 344-353, 1989.

KOTLER, P.; KELLER, K. L. Administração de marketing. 12. ed. São Paulo: Pearson Prentice Hall, 2006.

LILJANDER, V.; POLSA, P.; VAN RIEL, A. Modelling consumer responses to an apparel store brand: Store images as a risk reducer. Journal of Retailing and Consumer Services, v. 16, n. 4, p. 281-290, 2009.

MALHOTRA, N. K. Pesquisa de marketing: uma orientação aplicada. Porto Alegre: Bookman, 2006.

MATTAR, F. N. Pesquisa de marketing. São Paulo: Atlas, 2001.

MYERS, J. G. Determination of private brand attitudes. Journal of Marketing Research, v. 4, n. 1, p. 73-81, 1967.

OLIVER, R. L. Satisfaction: a behavioral perspective on the consumer. New York: McGraw-Hill, 1997. 
OLSON, J. C. Price as an informational cue: effects on product evaluations. In: WOODSIDE, A. G.; SHETH, J.N.; BENNETT, P. D. (Ed.). Consumer and industrial buying behavior. New York: Elsevier, 1977. p. 267-286.

PAULINS, V. A.; GEISTFELD, L. V. The effect of consumer perceptions of store attributes on apparel store preference. Journal of Fashion Marketing and Management, v. 7, n. 4, p. 371-385, 2003.

QUELCH, J. A.; HARDING, D. Brands versus private label: fighting to win. Harvard Business Review, v. 74, n. 1, p. 99-109, 1996.

RAO, A. R.; MONROE, K. B. The effect of price. Brand name and store name on buyers' perceptions of product quality: an integrated review. Journal of Marketing Research, v. 26, p. 351-357, 1989.

SCHINDLER, R. M.; HOLBROOK, M. M. Critical periods in men's and women's tastes in personal appearance. Psychology and Marketing, v. 10, n. 6, p. 549-564, 1993.

SHOCKER, A. D.; WEITZ, B. A. Perspective on brand equity principles and issues: in defining, measuring, and managing brand equity. Report No. 88-104. Ed. Lance, 1988.

SMITH, D. C. Brand Extension and advertising efficiency: What can and cannot be expected. Journal of Advertising Research, v. 32, p. 1120, 1992.

SNIPES, R. L; THOMSON, N. F.; OSWALD, S. L. Gender bias in customer evaluation of service quality: An empirical investigation. Journal of Services Marketing, v. 20, n. 4, p. 274-284, 2006.

SRIVASTAVA, R. K.; SHOCKER, A. D. Brand equity: a perspective on Its meaning and measurement. Cambridge: Marketing Science Institute, 1991. Working paper, p. 91-124.

SWINKER, M. E.; HINES, J. D. Understanding consumers' perception of clothing quality: a multidimensional approach, International Journal of Consumer Studies, v. 30, n. 2, p. 218-223, 2006. 
TYNAN, A. C.; DRAYTON, J. Market segmentation. Journal of Marketing Management, v. 2 , n. 3, p. 301-335, 1987.

WALSH, G.; MITCHELL, V.-W. Demographic characteristics of consumers who find it difficult to decide. Marketing Intelligence and Planning, v. 23, n. 2/3, p. 281-295, 2005.

WEDEL, M.; KAMAKURA, W. A. Market segmentation: conceptual and methodological foundations. $2^{\text {nd }}$. ed. Boston: Kluwer Academic Publishers, 2000.

YOO, B.; DONTHU, N. Developing and validating a multidimensional consumer-based brand equity scale. Journal of Business Research, v. 52, n. 1, p. 1-14, 2001.

YOO, B.; DONTHU, N.; LEE, S. An examination of selected marketing mix elements and brand equity. Journal of Academy of Marketing Science, v. 28, n. 2, p. 195-211, 2000.

ZEITHAML, V. A. Consumer perceptions of price, quality, and value: a means-end model and synthesis of evidence. Journal of Marketing, v. 52, n. 3, p. 2-22, 1988.

Artigo recebido em: 04/04/2013

Aprovado em: 31/10/2013 